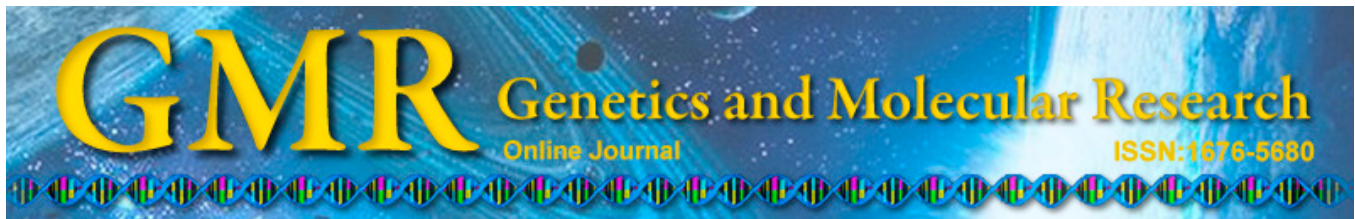

\title{
Characterization of the male-specific lethal 3 gene in the oriental river prawn, Macrobrachium nipponense
}

\author{
Y.P. Zhang', S.M. Sun ${ }^{2}$, H.T. Fu' ${ }^{2}$ X.P. Ge' ${ }^{2}$ H. Qiao', W.Y. Zhang ${ }^{2}$, \\ Y.W. Xiong' ${ }^{2}$, S.F. Jiang ${ }^{2}$, Y.S. Gong ${ }^{2}$ and S.B. Jin ${ }^{2}$ \\ ${ }^{1}$ Jiangxi Fisheries Research Institute, Nanchang, China \\ ${ }^{2}$ Key Laboratory of Freshwater Fisheries and Germplasm Resources Utilization, \\ Ministry of Agriculture, Freshwater Fisheries Research Center, \\ Chinese Academy of Fishery Sciences, Wuxi, China \\ Corresponding author: H.T. Fu \\ E-mail: fuht $@$ ffrc.cn
}

Genet. Mol. Res. 14 (2): 3106-3120 (2015)

Received May 9, 2014

Accepted September 24, 2014

Published April 10, 2015

DOI http://dx.doi.org/10.4238/2015.April.10.21

\begin{abstract}
In this study, male-specific lethal 3 homolog (Mnmsl3) was cloned and characterized from the freshwater prawn Macrobrachium nipponense (Crustacea: Decapoda: Palaemonidae) by rapid amplification of cDNA ends. The deduced amino acid sequences of Mnmsl3 showed high-sequence homology to the insect $M s l 3$ and contained a conserved chromatin organization modifier domain and an MORF4-related gene domain. Real-time quantitative reverse transcription-polymerase chain reaction showed that the $\mathrm{Mnmsl3}$ gene was expressed in all the investigated tissues, with the highest level of expression in the testis. The expression level of Mnmsl3 between males and females was different in the gonad (testis or ovary), abdominal ganglion, and heart. The results revealed that the Mnmsl3 gene might play roles in regulating chromatin and in dosage compensation of $M$. nipponense. Real-time quantitative reverse transcription-polymerase chain reaction also revealed that Mnmsl3 mRNA expression was
\end{abstract}


significantly increased in both 5 and 20 days post-larvae after metamorphosis, suggesting that $M n m s l 3$ plays complex and important roles in the early embryonic development and sex differentiation of $M$. nipponense.

Key words: Developmental expression; Macrobrachium nipponense; Crustacean; Male-specific lethal 3

\section{INTRODUCTION}

The dosage compensation effect, which occurs widely in eukaryotes that utilize sexual reproduction, is an essential biological process that equalizes the level of gene expression between genders based on sex determination (Sun et al., 2012). In Drosophila melanogaster, dosage compensation is an essential chromatin-mediated process and is required in males to up-regulate the single $\mathrm{X}$ chromosome by 2-fold to match the gene expression of the $2 \mathrm{X}$ chromosomes in females (Sural et al., 2008). The male-specific lethal (Msl) complex of D. melanogaster up-regulates the transcription of most genes on the male $\mathrm{X}$ chromosome, equalizing male and female X-linked gene expression (Bashaw and Baker, 1996; Lucchesi, 1998). The members of this gene family have been examined in previous studies, including Msll (Palmer et al., 1993), Msl2 (Zhou et al., 1995), Msl3 (Gorman et al., 1995), Mle (maleless) (Kuroda et al., 1991), Mof (males absent on first), and 2 noncoding RNAs (orX1 and orX2). Numerous studies have demonstrated that each $M s l$ protein is required for dosage compensation and male viability. Mutations in these genes, Mle, Msl1, Msl2, and Msl3, result in the death of homozygous males as third-instar larvae or early pupae because of the failure in dosage compensation (Belote and Lucchesi, 1980a; Okuno et al., 1984; Breen and Lucchesi, 1985; Gelbart and Kuroda, 2009; Gelbart et al., 2009). This illustrates that Msl gene plays an important role in dosage compensation mechanisms (Lucchesi et al., 2005).

In D. melanogaster, $M s l 3$ is a chromodomain protein with RNA-binding activity in vitro (Jones et al., 2000; Buscaino et al., 2006). This protein is essential for the activation of Mof nucleosomal histone acetyl transferase activity in the Msll-Mof complex (Morales, 2005). Msl3 is likely to be involved in chromatin remodeling and transcriptional regulation and is associated with hundreds of specific sites along the length of the male X chromosome, but not the female X chromosome (Gorman et al., 1995). Gorman suggested that Msl3 also functions as a direct mediator of dosage compensation. The main components of mammalian and fish Msl complex have already been identified (Smith et al., 2000). However, little is known regarding the developmental expression analysis of the male-specific lethal 3 gene in crustaceans. Because crustaceans have a very close evolutionary relationship with insects (Budd and Telford, 2009), we hypothesized that the homolog Mnmsl3 from oriental river prawn Macrobrachium nipponense is involved in regulating sex determination in crustaceans.

We identified a homologous sequence fragment of Msl3 from the testis cDNA library of M. nipponense (Qiao et al., 2012), which is a commercial freshwater prawn. It is considered an important fishery resource in China, with an annual production of 205,010 tons (Bureau of Fishery, 2009). The molecular mechanisms regulating the expression of sex-specific and sexdetermining genes in M. nipponense have been examined previously in our lab (Zhang et al., 2013). In the present study, we cloned the cDNA encoding the Mnmsl3 gene from the cDNA library of testis in M. nipponense and examined the expression pattern of the Mnmsl3 gene 
during the embryonic development, larvae, and post-larvae stages. This information provides insightful directions for future research on the mechanisms of sex determination and dosage compensation in the oriental river prawn.

\section{MATERIAL AND METHODS}

\section{Embryo, larvae, and tissue collections}

Healthy adult oriental river prawns with wet weights of 2.26-6.25 g were obtained from Tai Lake in Wuxi, China $\left(120^{\circ} 13^{\prime} 44^{\prime \prime E}, 31^{\circ} 28^{\prime} 22^{\prime \prime} \mathrm{N}\right)$. All samples were transferred to laboratory breeding conditions and maintained in a 500-L tank with aerated freshwater for $72 \mathrm{~h}$ before tissue collection. Embryos and larvae in different developmental stages were obtained from our breeding room. After prawn spawning, each developmental stage of the embryos (cleavage stage; blastula stage; gastrula stage; nauplius stage; protozoea stage; zoea stage) was collected according to the morphological methods following the criteria of Chen et al. (2012). Larvae were collected every 3 days from 1 day post-hatching (L1) to L13 (1 day before metamorphosis). Post-larvae were collected every 4 days from 1-20 days after metamorphosis (P1-P20), and every 10 days from P20-P30. The ovary was collected from mature female prawns, but the testis, muscle, heart, abdominal ganglion, brain, and intestine were collected from mature male prawns. The samples were washed with $1 \mathrm{X} 0.01 \mathrm{M}$ phosphatebuffered saline, frozen in liquid nitrogen, and stored at $-80^{\circ} \mathrm{C}$ until processing.

\section{Histological analysis}

The water temperature was $28^{\circ} \mathrm{C}$. Between 5 and 10 post-larvae were collected every 2 days between 1-15 days after metamorphosis, every 5 days between 15-30 days, and every 5 days between 30-35 days. After anesthetization with Eugenol $(100 \mathrm{mg} / \mathrm{L})$, the total length (TL) and body weight (BW) of each prawn were measured to the nearest $0.01 \mathrm{~mm}$ and 0.0001 g. For prawns $\leq 10 \mathrm{~mm}$ TL, the whole body was fixed with Bouin's solution at room temperature for at least $24 \mathrm{~h}$, washed in 50\% ethanol, and stored in $70 \%$ ethanol until histological processing. For specimens larger than $10 \mathrm{~mm}$ TL, the abdominal segments and brow was removed, and the carapace was fixed with Bouin's solution. Tissues were dehydrated in a series of alcohol, clarified in benzene, and embedded in paraffin. Cross-sections were cut every 5-7 $\mu \mathrm{m}$ and were stained with Mayer's hematoxylin and eosin phloxine B solution following conventional histological procedures (Park et al., 1998). A thorough examination of gonads was performed by cutting the prawn's whole carapace into serial sections. The use of serial sections was found to be important for determining the developmental stage of the gonads. The slides were examined and photographed under a light microscope with a video camera linked to the computer image analysis software (Olympus, Tokyo, Japan).

\section{RNA isolation and reverse transcription-polymerase chain reaction (RT-PCR)}

Total RNA was extracted from embryos and larvae at different stages of development and from other tissues in mature prawns with RNAiso Plus Reagent (TaKaRa Bio Inc., Shiga, Japan) in accordance with the manufacturer protocol. Isolated RNA was treated with RNasefree DNase I (Sangon, Shanghai, China) to eliminate genomic DNA contamination. The con- 
centration of each total RNA sample was then measured using a BioPhotometer (Eppendorf, Hamburg, Germany), and $2 \mu \mathrm{L}$ was analyzed on a $1 \%$ agarose gel to evaluate the integrity. cDNA was synthesized from $5 \mu \mathrm{g}$ total RNA using the PrimeScript ${ }^{\mathrm{TM}}$ RT-PCR Kit (TaKaRa) according to manufacturer protocols. The cDNA was stored at $-20^{\circ} \mathrm{C}$ until real-time quantitative RT-qPCR.

\section{5'- and 3'-random amplification of cDNA ends (RACE) of the Msl3 gene}

Four gene-specific primer sets (Table 1) were designed based on the expressed sequence tag of male-specific lethal 3 homolog (GenBank accession No. JK525590.1) obtained from the M. nipponense testis cDNA library (Qiao et al., 2012). The cloning of Mnmsl3 cDNA, 3'- and 5'-RACE cDNA was performed using the 3'-full RACE Core Set Ver. 2.0 Kit and 5'full RACE Kit (TaKaRa) to transcribe according to the manufacturer protocol, respectively. For 5'-RACE, the primer sets consisted of 2 gene-specific primers of GSP1 and GSP2 and the universal primers 5'-RACE OUT, and 5'-RACE IN (Table 1). For 3'-RACE, the primer sets consisted of 2 gene-specific primers for GSP3 and GSP4 and the universal primers 3'-RACE OUT and 3'-RACE IN (Table 1).

\begin{tabular}{lll}
\multicolumn{2}{l}{ Table 1. Nucleotide sequences of primers used for Mnmsl3 cloning and expression analysis. } & \\
\hline Name & Sequence $\left(5^{\prime} \rightarrow 3^{\prime}\right)$ & Code \\
\hline Primers for 5'-RACE PCR & & GSP1 \\
Mnmsl1 5'-GSP primer 1 & CACCTCTGAGGCCCCTGACCC & GSP2 \\
Mnmsl1 5'-GSP primer 2 & AGCAAAGCACACGCTCACCCTC & GSP3 \\
Primers for 3'-RACE PCR & & GSP4 \\
Mnmsl1 3'-GSP primer 1 & ACCGAGTACTCCTTTGGGGC & \\
Mnmsl1 3'-GSP primer 2 & CATTCCTCACCCTGCACCTT & \\
Full RACE'M Kit primers & TACCGTCGTTCCACTAGTGATTT & \\
3'-RACE OUT & CGCGGATCCTCCACTAGTGATTTCACTATAGG & \\
3'-RACE IN & CATGGCTACATGCTGACAGCCTA & \\
5'-RACE OUT & CGCGGATCCACAGCCTACTGATGATCAGTCGATG & RT-F1 \\
5'-RACE IN & & RT-R1 \\
Primers for real-time PCR analysis & GTTGCAGCGTCAACTAGCAG & $\beta$-actinF \\
Mnmsl1 5'-primer & AAGTCTACGTTCCTGCGCT & -actinR \\
Mnmsl1 3'-primer & TATGCACTTCCTCATGCCATC & -actin 5'-primer \\
$\beta$-actin 3'-primer & AGGAGGCGGCAGTGGTCAT & \\
\hline
\end{tabular}

\section{Primer sequences}

PCR products were gel-purified and ligated into the pMD18-T vector (TaKaRa) following manufacturer instructions. The recombinant vectors were then transformed into Escherichia coli DH5a (Qiagen, Hilden, Germany) competent cells; 5 were identified by blue/ white screening and confirmed by PCR. At least 5 positive clones were sequenced in both directions using an automatic DNA sequencer (Applied Biosystems ABI-3730, Foster City, CA, USA). The resulting sequences were verified and subjected to cluster analysis using the online database of the National Center for Biotechnology Information.

\section{Nucleotide sequence and bioinformatic analyses}

The 5'- and 3'-sequences from RACE were assembled with the partial cDNA sequence 
corresponding to each fragmental sequence using DNAMAN 5.0. Sequences were analyzed based on the nucleotide and protein databases using the BLASTX and BLASTN programs (http://www.ncbi.nlm.nih.gov/BLAST/). Protein prediction was performed using the ORF finder (http://www.ncbi.nlm.nih.gov/gorf/). The ProtParam program (http://www.expasy. $\mathrm{ch} /$ tools/protparam.html) was used to compute physical and chemical parameters of the amino acid sequence. The motif was searched using the motif scan program (http://myhits. isb-sib.ch/cgi-bin/motif_scan). Multiple alignments of $M s l 3$ (Table 2) were generated using the ClustalW1.81 program and obtained alignments were used to construct phylogenetic trees using the neighbor-joining method. The bootstrapping test was performed using 1000 replications with Molecular Evolutionary Genetics Analysis, MEGA4 (Tamura et al., 2007).

Table 2. $M s l 3$ information used for sequence alignment and phylogenic analysis.

\begin{tabular}{|c|c|c|}
\hline Name & Accession No. & Organism \\
\hline B. impatiens $\mathrm{Ms} 13$ & XP_003488824.1 & Bombus impatiens \\
\hline A. florea $\mathrm{Msl} 3$ & XP_003697744.1 & Apis florea \\
\hline M. nipponense $\mathrm{Msl} 3$ & KC̄793887 & Macrobrachium nipponense \\
\hline D. plexippus $\mathrm{Ms} 13$ & EHJ74368.1 & Danaus plexippus \\
\hline H. saltator $\mathrm{Msl} 3$ & EFN82979.1 & Harpegnathos saltator \\
\hline C. floridanus Msl3 & EFN67569.1 & Camponotus floridanus \\
\hline A. echinatior Msl3 & EGI57386.1 & Acromyrmex echinatior \\
\hline M. rotundata $\mathrm{Msl} 3$ & XP_003704853.1 & Megachile rotundata \\
\hline C. clemensi $\mathrm{Msl} 3$ & ACO15424.1 & Caligus clemensi \\
\hline C. quinquefasciatus $\mathrm{Msl} 3$ & EDS28869.1 & Culex quinquefasciatus \\
\hline M. musculus $\mathrm{Ms} 13$ & NP_034962.2 & Mus musculus \\
\hline M. occidentalis Msl3 & XP_003741273.1 & Metaseiulus occidentalis \\
\hline D. melanogaster $\mathrm{Ms} 13$ & NP_523951.1 & Drosophila melanogaster \\
\hline B. mori $\mathrm{Msl} 3$ & NP_001093308.1 & Bombyx mori \\
\hline
\end{tabular}

\section{Real-time RT-qPCR analysis of $M s l 3$}

The Mnmsl3 mRNA expression at different stages, from embryo to post-larval, and in various adult tissues were measured using SYBR Green real-time RT-qPCR analysis in the Bio-Rad iCycler iQ5 Real Time System (Bio-Rad, Hercules, CA, USA). Gene-specific primers (Table 1) were used to amplify the Mnmsl3 transcript, and the PCR products were sequenced to verify the specificity of the PCR primers. Three samples were collected from each developmental stage and each tissue. Each sample was analyzed in triplicate with each reaction well containing $25 \mu \mathrm{L}$ PCR mixture composed of $1 \mu \mathrm{L}$ cDNA (50 ng), $10 \mu \mathrm{L}$ SsoFast $^{\mathrm{TM}}$ EvaGreen $^{\circledR}$ Supermix (Bio-Rad), $0.5 \mu \mathrm{L} 10 \mu \mathrm{M}$ gene-specific forward and reverse primers (Table 1), and $13 \mu \mathrm{L}$ diethylpyrocarbonate-water. The reaction mixture was initially incubated for $30 \mathrm{~s}$ at $95^{\circ} \mathrm{C}$ to activate the Hot Start Taq DNA polymerase, followed by 40 cycles of $10 \mathrm{~s}$ denaturation at $95^{\circ} \mathrm{C}$ and a $10 \mathrm{~s}$ extension at $60^{\circ} \mathrm{C}$. Melting curve analysis was performed over a range of $65^{\circ}-95^{\circ} \mathrm{C}$ (in $0.5^{\circ} \mathrm{C}$ increments) for $10 \mathrm{~s}$ to verify the generation of a single product. Amplification of $\beta$-actin (Zhang et al., 2013) as an internal reference was also carried out in the same sample (primer sequences are shown in Table 1). As a negative control, diethylpyrocarbonate-water replaced the template. The relative copy number of Mnmsl3 mRNA was calculated according to the $2^{-\Delta \Delta C t}$ comparative CT method (Livak and Schmittgen, 2001). 


\section{Statistical analysis}

All data are reported as means \pm standard error of the mean $(\mathrm{N}=3)$. Statistical analysis was performed using the SPSS 13.0 software (SPSS, Inc., Chicago, IL, USA). Statistical significance was determined using one-way analysis of variance and post hoc Duncan multiple range tests. Significance was set at $\mathrm{P}<0.05$.

\section{RESULTS}

\section{Molecular cloning and identification of $\mathrm{Mnmsl3}$ cDNA}

The full-length cDNA sequence of Mnmsl3 was $1378 \mathrm{bp}$, and contained an open reading frame of $1092 \mathrm{bp}$, an untranslated region of $215 \mathrm{bp}$ the 5'-end, a 3'-untranslated region of $71 \mathrm{bp}$, and a complete poly A tail. The A+T content and $\mathrm{C}+\mathrm{G}$ content in the sequence were 60.1 and $39.9 \%$, respectively. Analysis of the deduced protein sequence of the Msl3 showed that it was comprised of 363 amino acids with a predicted molecular mass of $42.493 \mathrm{kDa}$ and isoelectric point of 5.11. This cDNA sequence was submitted to GenBank under accession No. KC793887. The conserved sequence and characteristic motifs of the chromatin organization modifier domain, chromo-barrel domain, and MRG were identified in the deduced amino acid sequences of Mnmsl3, and the conserved motifs were located in the N-terminal region and central region, respectively (Figure 1).

Protein functional sites in Mnmsl3 were predicted using ExPASy. The mature protein was composed of 7 protein kinase $\mathrm{C}$ phosphorylation sites, 12 casein kinase II phosphorylation sites, 2 tyrosine kinase phosphorylation sites, 2 cAMP- and cGMP-dependent protein kinase sites, 1 amidation site, $3 \mathrm{~N}$-myristoylation sites, and $2 \mathrm{~N}$-glycosylation sites (Table 3).

\section{Homology and phylogenetic analysis of $\mathrm{Mnmsl3}$}

The amino acid sequence of Mnmsl3 was compared with the sequences of previously reported Msl3 proteins. The Mnmsl3 protein shared high identity with those of other species, including Bombus impatiens (XP_003488824.1; 43\%), Apis florea (XP_003697744.1; 42\%), Danaus plexippus (EHJ74368.1; $42 \%$ ), and Bombyx mori (NP_001093308.1; 40\%) (Figure 2). A neighbor-joining phylogenic tree was constructed based on reported arthropod Msl3 amino acid sequences using the MEGA 4.0 software. The tree showed that Mnmsl3 was not present in crustaceans but was present in Hymenoptera (Figure 3), suggesting a closer phylogenetic relationship with Hymenoptera.

\section{Tissue distribution of $M n m s l 3$ mRNA}

The expression patterns of Mnmsl3 in the testis, ovary, heart, abdominal ganglion, brain, and muscle tissues were also examined. RT-qPCR analysis of Mnmsl3 mRNA indicated that it was expressed in all examined tissues of adult prawn, with the highest expression in the testis, followed by the brain. The lowest level was detected in the muscle tissue (Figure 4). In addition, we observed sexually dimorphic expression of Mnxsl3 in male and female prawns; Mnmsl3 mRNA expression was higher in the testis, heart, and abdominal ganglion in males compared to female prawns $(\mathrm{P}<0.05$; Figure 5). 


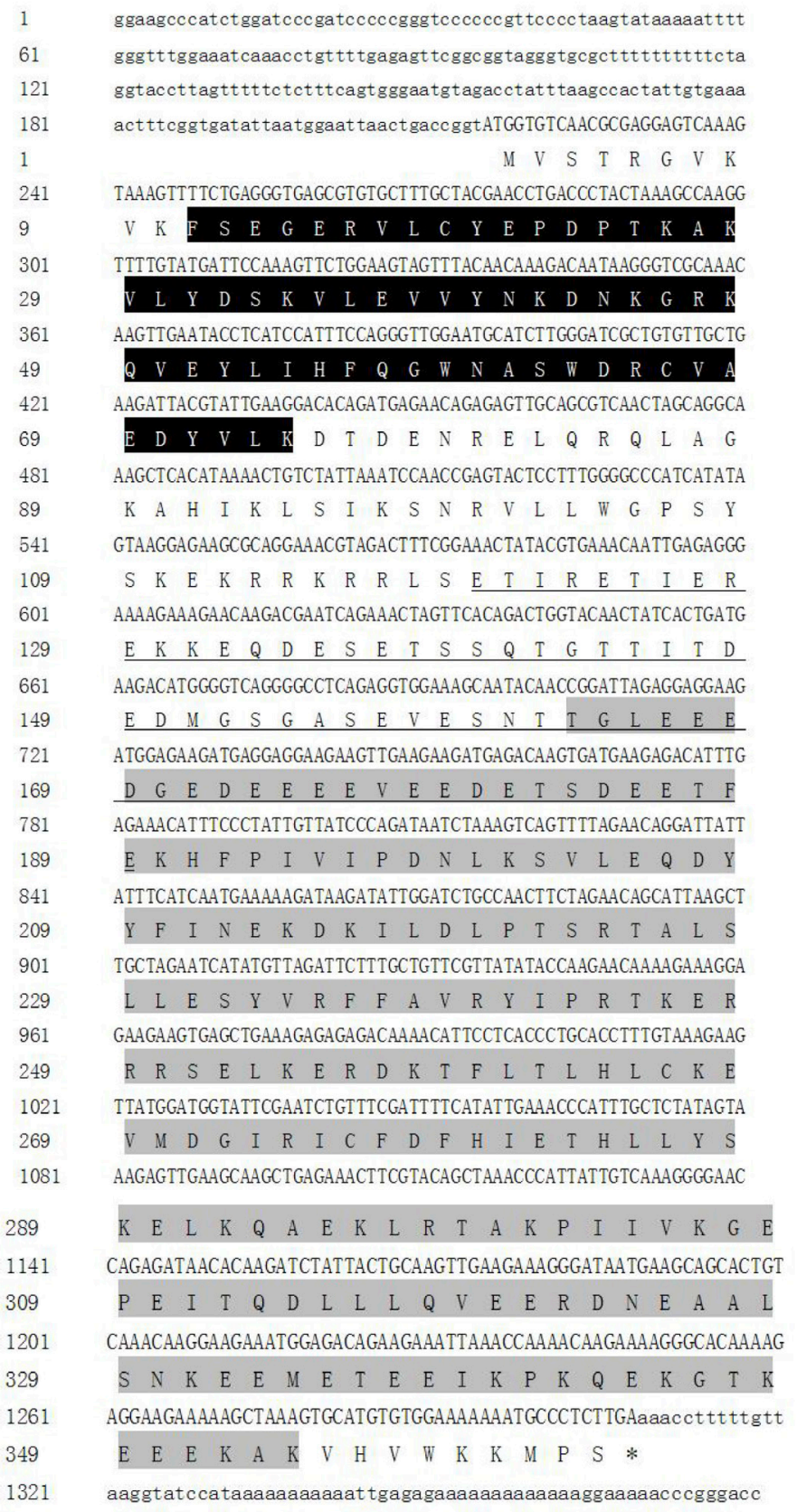

Figure 1. cDNA and deduced amino acid sequences of Mnmsl3. cDNA sequence is numbered from the first nucleotide. Deduced amino acid sequence is bold and numbered relative to the first residue, and is shown in singleletter code below the relevant nucleotide sequence. The nucleotide sequences encoding the start codon (ATG) and stop codon (TGA) are bolded and the stop codon is underlined by a single line, with an asterisk below. The glutamic acid-rich region is indicated with a black single line. The chromo and MRG domains are marked with black and shadow, respectively. 
Table 3. Functional site analysis of the mature Mnmsl3 protein.

\begin{tabular}{ll}
\hline Function site & AA position \\
\hline Protein kinase C phosphorylation site & $3-5$ (STR), 95-97 (SIK), 98-100 (SNR), 121-123 (TIR), 222-224 (TSR), \\
Casein kinase II phosphorylation site & 299-301 (TAK), 329-331 (SNK) \\
& $12-15$ (SEGE), 121-124 (TIRE), 145-148 (TITD), 147-150 (TDED), \\
& $202-205$ (SEVE), 163-166 (TGLE), 182-185 (TSDE), 183-186 (SDEE), \\
Amidation site & $45-48$ (KGRK) \\
Tyrosine kinase phosphorylation site & $34-40$ (kvl.evv.y), 201-209 (KSVLEQDYY) 329-332 (SNKE), -347-350 (TKEE) \\
N-myristoylation site & $143-148$ (GTTITD), 152-157 (GSGASE), 272-277 (GIRICF) \\
N-glycosylation site & $60-63$ (NASW), 161-164 (NTTG) \\
cAMP- and cGMP-dependent protein kinase & $116-119$ (RRLS), 248-251 (RRRS) \\
\hline
\end{tabular}

\section{Expression analysis of Mnmsl3 mRNA during embryo, larvae, and post-larval stages}

Mnmsl3 mRNA expression levels were examined using real-time RT-qPCR on embryos at different developmental stages, including the larval and post-larval stages. The results revealed that the Mnmsl3 gene was expressed in all developmental stages of M. nipponense. The expression level of Mnmsl3 increased gradually from the cleavage stage to the nauplius stage, and was highest during the nauplius stage. Subsequently, expression gradually declined with the development of the embryo and larvae. The lowest expression levels of the Mnmsl3 were found at L13, as well as 1 day before metamorphosis (Figure 6). After metamorphosis, the expression of Mnmsl3 mRNA abruptly increased from P1-P5 and peaked at P5 (Figure 6), and then abruptly decreased at P10. Subsequently, the Mnmsl3 level increased again from P15-20, but also abruptly declined at P25 (Figure 6).

\section{Sex differentiation and development}

Based on histological observation, the ovaries and testes of $M$. nipponense developed directly from undifferentiated gonadal tissue, and thus $M$. nipponense can be classified as a differentiated gonochorist.

The result showed that the primordial germ cells were observed in M. nipponense on the 11th day after metamorphosis (Figure 7a), with primordial germ cells differentiating into germ cells on the 15th day (Figure 7b). The time of ovarian differentiation was earlier than that of the testis, and the differentiation pattern differed between the ovary and testis. Ovarian differentiation was initiated on the 18th day after metamorphosis (Figure $7 \mathrm{c}$ ) and the ovarian cavity formed on the 30th day (Figure 7d). The testis began to differentiate on the 20th day after metamorphosis (Figure 7e), with the seminiferous tubule formed and filled with germ cells at 23-25 days after metamorphosis (Figure 7f-g). Testis differentiation was completed by the 30th day after metamorphosis (Figure $7 \mathrm{~h}$ ). These findings indicate that the time to ovarian differentiation was earlier than that of testis differentiation. 


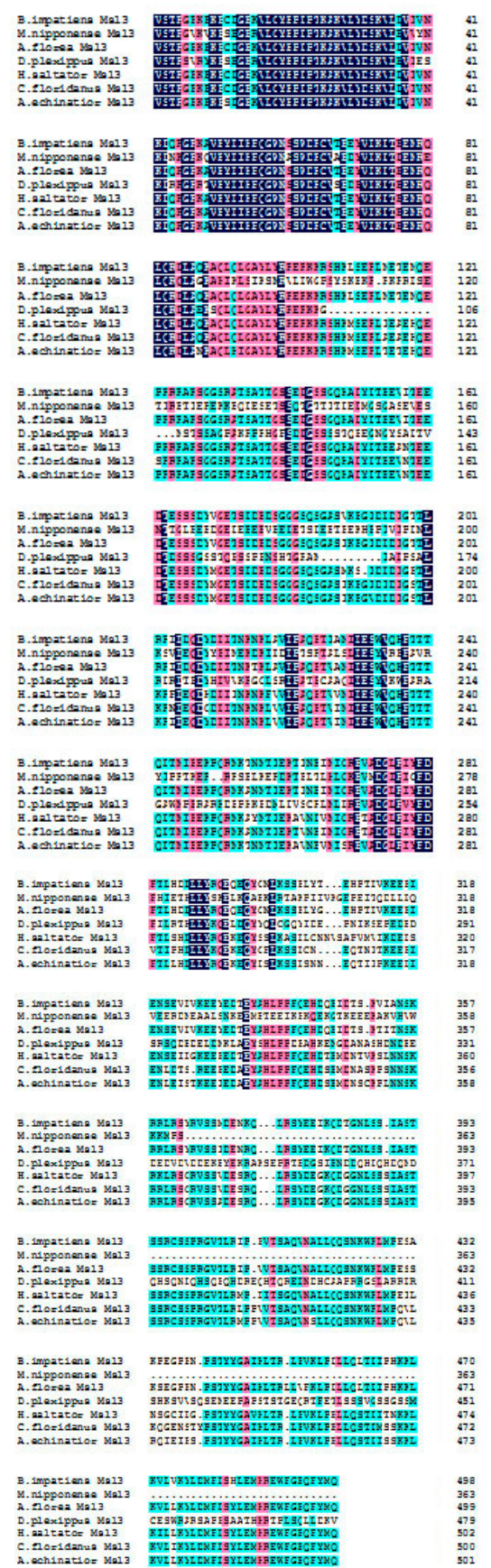

Figure 2. Multiple alignment of Macrobrachium nipponense Msl3 sequence with that from other species using DNAMAN. The deduced amino acid sequences are summarized in Table 2. Identical amino acid residues are highlighted in dark and similar amino acids are highlighted in pink or green. Inserts (periods) are added to maximize sequence identity. 


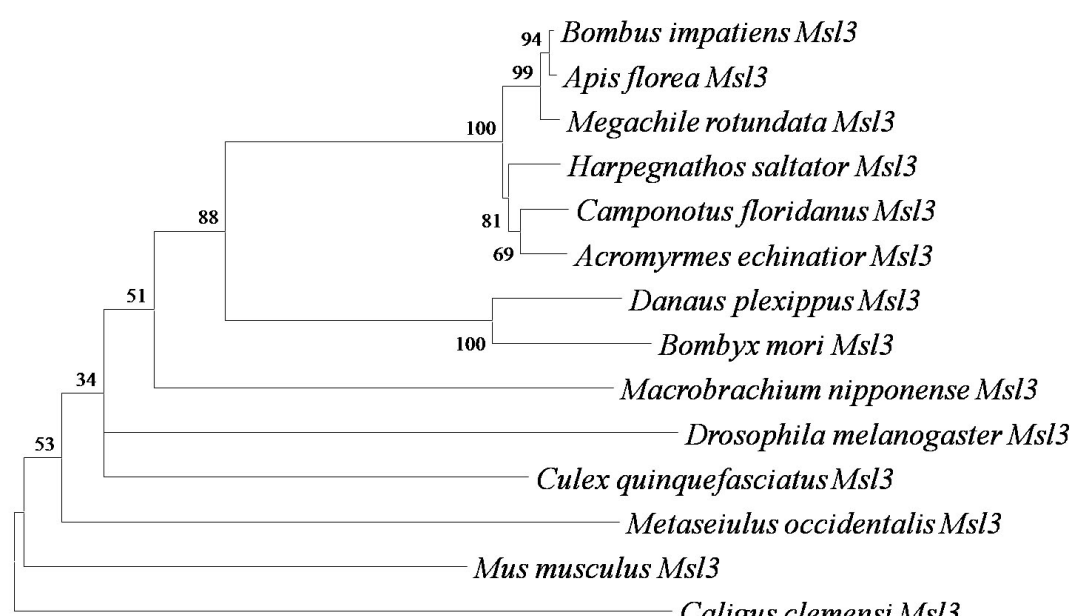

Caligus clemensi Msl3

0.1

Figure 3. Neighbor-joining phylogenetic tree of $M n m s l 3$ and $M s l 3$ homologs from other species. The sequences used in the phylogenetic tree are summarized in Table 3. Bootstrap analysis of 1000 replicates was carried out to determine the confidence of tree branch positions. The numbers marked on the tree branches represent the bootstrap values.

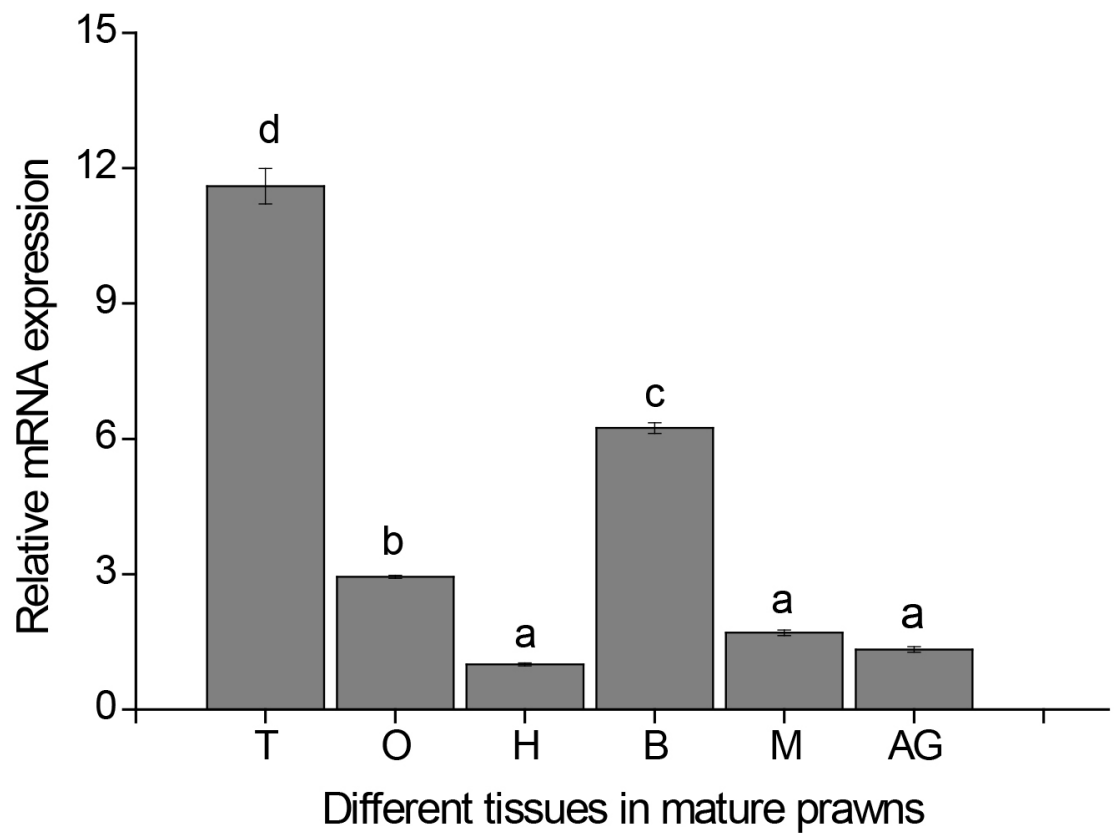

Figure 4. Expression characterization of $\mathrm{Mnmsl3}$ in the various adult tissues was revealed by real-time quantitative PCR. The amount of Mnmsl3 mRNA was normalized to the $\beta$-actin transcript level. Data are reported as means \pm standard error of the mean of 3 separate individuals in the tissues. Bars with different letters were considered significant at $\mathrm{P}<0.05 . \mathrm{T}=$ testis; $\mathrm{O}=$ ovary; $\mathrm{B}=$ brain; $\mathrm{AG}=$ abdominal ganglion; $\mathrm{H}=$ heart; $\mathrm{M}=$ muscle. 


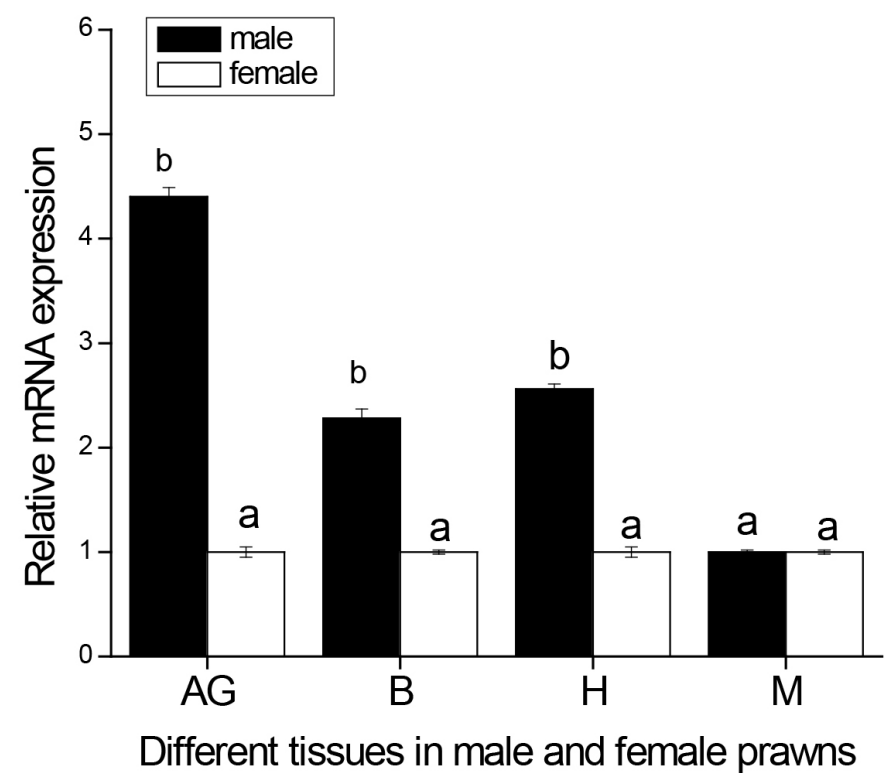

Figure 5. Distribution of Msl3 mRNA in the different tissues of male and female prawns. Data are reported as mean fold-change (means \pm standard error of the mean, $\mathrm{N}=3$ ). Statistical significance was calculated by one-way analysis of variance and multiple comparison tests.

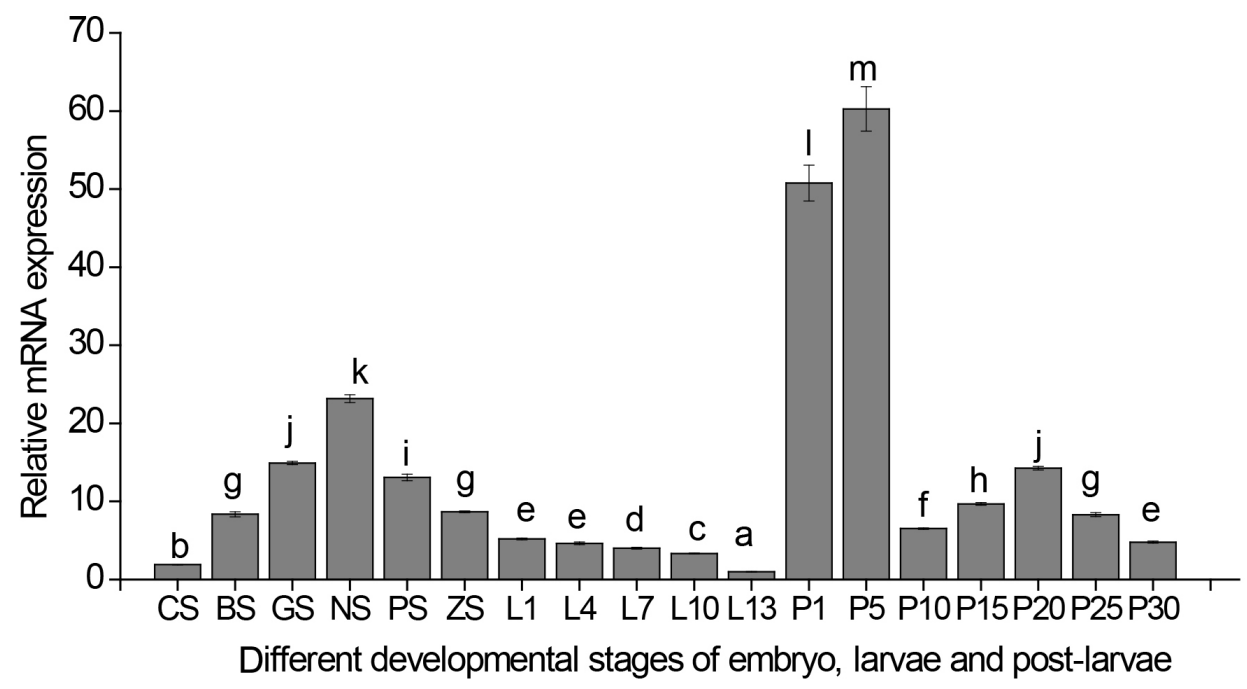

Figure 6. Temporal expression of Mnmsl3 during the different development stages of embryos, larvae before metamorphosis, and post-larvae after metamorphosis was revealed by real-time quantitative PCR. The amount of Mnmsl3 mRNA was normalized to the $\beta$-actin transcript level. Data are reported as means \pm standard error of the mean of 3 repeated samples during the embryo, larva, and post-larva stages. Bars with different letters were considered significant at $\mathrm{P}<0.05$. $\mathrm{CS}=$ cleavage stage; $\mathrm{BS}=$ blastula stage; $\mathrm{GS}=$ gastrula stage; $\mathrm{NS}=$ nauplius stage; $\mathrm{PS}=$ protozoea stage; $\mathrm{ZS}$ = zoea stage. $\mathrm{L1}$ = first day larva after hatching, and so on; $\mathrm{P} 1$ = first day post-larva after metamorphosis, and so on. 

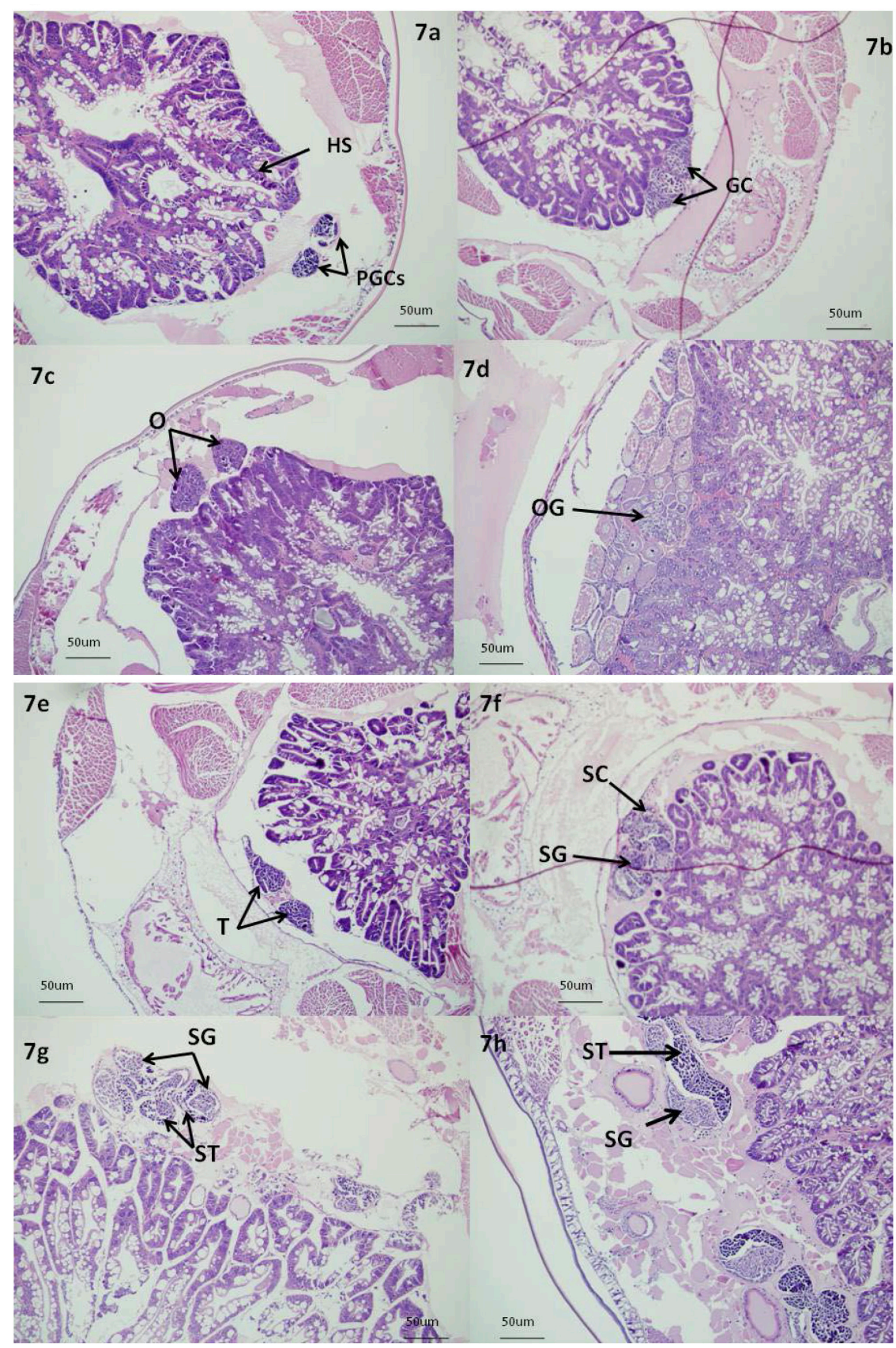

Figure 7. Plate gonad histological sections of Macrobrachium nipponense. a. Cross-section of gonad at 11 days, 100X; b. cross-section of gonad at 15 days, 100X; c. cross-section of gonad at 18 days, $100 \mathrm{X}$; d. cross-section of gonad at 30 days, 100X; e. cross-section of gonad at 20 days, 100X; f. cross-section of gonad at 23 days (testis), 100X; g. crosssection of gonad at 25 days, $100 \mathrm{X} ; \mathbf{h}$. cross-section of gonad at 30 days, $100 \mathrm{X}$. HS = hepatopancreas; PGCs = primordial germ cells; $\mathrm{GC}=$ germ cell; $\mathrm{SG}=$ spermatogonia; $\mathrm{SC}=$ Sertoli cell; $\mathrm{ST}=$ seminiferous tubules; $\mathrm{SC}=$ spermatocyte. 


\section{DISCUSSION}

In Drosophila, the Msl complex selectively binds the male X chromosome and is necessary for both chromosome puffing and equalization of transcript levels (Laverty et al., 2010). Gorman et al. (1995) found that Msl3 is also bound to the male $\mathrm{X}$ chromosome. The $M s l 3$ gene is part of a small gene family that is widespread in eukaryotes, and all eukaryotic cells may contain Msl3 analogs. In the present study, we cloned and identified Msl3 homologs in M. nipponense referred to as Mnmsl3. The Msl3 homologs in insect species contain 2 highly conserved domains, including an N-terminal chromo domain (Koonin et al., 1995; Jones et al., 2002; Buscaino et al., 2006) and a C-terminal MRG (Marín and Baker, 2000) domain. The chromo domain is considered a site of protein-protein interactions. Additionally, it participates in the alteration of chromatin structure and positive-negative transcriptional regulation (Cavalli and Paro, 1998). In contrast, the MRG domain co-localizes with the $\mathrm{X}$ chromosome (Buscaino et al., 2006). In this study, by comparing insect sequences with the Mnmsl3 gene, we identified chromo-barrel domain regions in these proteins that showed a high degree of sequence conservation, suggesting that Mnmsl3 performs similar functions in regulating chromatin and in dosage compensation of M. nipponense. Phylogenetic analysis revealed that $M n m s l 3$ was closely related to Hymenoptera and Lepidoptera Msl3, but differed from their homologs in the crustacean Caligus clemensi. The exact orthologous relationship of Mnmsl3 requires further study, including the cloning of additional Msl3 from crustaceans.

In Drosophila, Msl3 is expressed in both sexes, including the liver, pancreas, heart, lung, kidney, skeletal muscle, brain, and placenta, with highest expression in skeletal muscle and heart, but is severely reduced in females (Gorman et al., 1995). We found that Mnmsl3 mRNA was also expressed in different tissues of M. nipponense, with the highest expression in the testis, followed by in the brain. The distribution of $M s l 3$ mRNA showed some differences between M. nipponense and Drosophila. This implied that Mnmsl3 might have other functions in M. nipponense that developed during evolution. In addition, we found that the expression of Mnmsl3 mRNA differed between the sexes; with the exception of muscle ( $\mathrm{P}>0.05)$, expression level in the testes, abdominal ganglia, brain, and heart in males being significantly higher than that in females $(\mathrm{P}<0.05)$. These data indicate that a mechanism for dosage compensation exists in male freshwater prawns, but this requires further study.

The developmental process of oocytes in the prawn exhibits a series of cellular differentiation process in which differential genes are expressed temporally and spatially to ensure the proper development of the oocytes (Qiu et al., 2005; Meeratana and Sobhon, 2007). In the present study, we found that Mnmsl3 mRNA was expressed during all stages, including the cleavage stage during embryonic development of M. nipponense. However, Mnmsl3 mRNA was mainly expressed in the developing embryo. The results of the present study showed that the expression of Mnmsl3 gradually increased with embryonic development, peaking during the nauplius stage. Because embryonic organogenesis of M. nipponense starts from the nauplius stage and is most active at the protozoea stage (Zhang et al., 2010), the expression of Mnmsl3 during embryogenesis and organogenesis in this study indicate that Mnmsl3 has roles in the embryonic development of M. nipponense. During the larval stage, Mnmsl3 mRNA expression gradually declined with larval age before metamorphosis, but decreased to the lowest level at the metamorphic climax in M. nipponense. The expression patterns of $\mathrm{Mnmsl3}$ during larval development may be necessary to stimulate 
pre-metamorphic larval changes of morphology and initiate metamorphosis. Previous studies have shown that sex differentiation and sexually dimorphic development of crustaceans begin after metamorphosis (Lee et al., 1994; Zhao et al., 2009). As demonstrated in a previous study, changes in external sexual forms occur before those in internal sexual forms (Zhu et al., 2011). On histological sections, primordial germ cells in M. nipponense appeared in 10-15 days post-larvae, with external sexual forms occurring at 5-10 days post-larvae. The time of Mnmsl3 action in post-larvae was generally correlated with the occurrence time of primordial germ cells and external sexual forms of M. nipponense, suggesting that Mnmsl3 homologs were involved in sex differentiation and determining external sexual forms in $M$. nipponense.

In conclusion, Mnmsl3 may play important roles in the embryonic development and in post-larval sex differentiation and external sexual formation of $M$. nipponense. This study increases the understanding of the multiple biological functions of the Mnmsl3 genes, and lays a foundation for studies examining the regulation mechanisms of the dosage compensation pathway in $M$. nipponense. In addition, based on histological observation of gonadal differentiation and development of $M$. nipponense, the critical period of sex differentiation in $M$. nipponense occurs between 10-15 days after metamorphosis when sexually undifferentiated gonads are more responsive to the action of exogenous steroids.

\section{ACKNOWLEDGMENTS}

Research supported by the National Natural Science Foundation of China (Grant \#31272654), the Freshwater Fisheries Research Center, China Central Governmental Research Institutional Basic Special Research Project from the Public Welfare Fund (\#2013JBFM15), the National Science \& Technology Supporting Program of the 12th Five-Year Plan of China (Grant \#2012BAD26B04), the Jiangsu Provincial Natural Science Foundation for Young Scholars of China (Grant \#BK2012091), the Special Fund for Agro-Scientific Research in the Public Interest (\#201303056-6), the Jiangxi Province Science and Technology Support Project (\#20133BBF60029), the Science \& Technology Supporting Program of Jiangsu Province (Grant \#BE2012334), and the 3 Aquatic Projects of Jiangsu Province (\#D2013-6).

\section{REFERENCES}

Bashaw GJ and Baker BS (1996). Dosage compensation and chromatin structure in Drosophila. Curr. Opin. Genet. Dev. 6: 496-501.

Belote JM and Lucchesi JC (1980a). Control of X chromosome transcription by the maleless gene in Drosophila. Nature 285: 573-574.

Breen TR and Lucchesi JC (1985). Analysis of the dosage compensation of a specific transcript in Drosophila melanogaster. Genetics 112: 483-491.

Budd GE and Telford MJ (2009). The origin and evolution of arthropods. Nature 457: 812-817.

Bureau of Fishery. Ministry of Agriculture, P.R.C. (2009). Fisheries Economic Statistics. China Agricultural Press, Beijing.

Buscaino A, Legube G and Akhtar A (2006). X-chromosome targeting and dosage compensation are mediated by distinct domains in MSL-3. EMBO Rep. 7: 531-538.

Cavalli G and Paro R (1998). Chromo-domain proteins: linking chromatin structure to epigenetic regulation. Curr. Opin. Cell Biol. 10: 354-360.

Chen Y, Zhu Q, Chen H, Zhu XL, et al. (2012). The morphological and histological observation of embryonic development in the oriental river prawn Macrobrachium nipponense. J. Shanghai Ocean Univ. 21: 33-40.

Gelbart ME and Kuroda MI (2009). Drosophila dosage compensation: a complex voyage to the X chromosome. Development 136: 1399-1410. 
Gelbart ME, Larschan E, Peng SY, Park PJ, et al. (2009). Drosophila MSL complex globally acetylates H4K16 on the male X chromosome for dosage compensation. Nat. Struct. Mol. Biol. 16: 825-832.

Gorman M, Franke A and Baker BS (1995). Molecular characterization of the male-specific lethal-3 gene and investigations of the regulation of dosage compensation in Drosophila. Development 121: 463-475.

Jones DO, Cowell IG and Singh PB (2002). Mammalian chromodomain proteins: their role in genome organisation and expression. Bioessays 22: 124-137.

Koonin EV, Zhou S and Lucchesi JC (1995). The chromo superfamily: new members, duplication of the chromo domain and possible role in delivering transcription regulators to chromatin. Nucleic Acids Res. 23: 4229-4233.

Kuroda MI, Keman MJ, Kreber R, Ganetsky B, et al. (1991). The maleless protein associates with the X chromosome to regulate dosage compensation in Drosophila. Cell 66: 935-947.

Laverty C, Lucci J and Akhtar A (2010). The MSL complex: X chromosome and beyond. Curr. Opin. Genet. Dev. 20: 171-178.

Lee TH, Yamauchi M and Yamazaki F (1994). Sex differentiation in the crab Eriocheir japonicas (Decapoda, Grapsidae). Invert. Reprod. Develop. 25: 123-138.

Livak KJ and Schmittgen TD (2001). Analysis of relative gene expression data using real-time quantitative PCR and the 2 (-Delta Delta C (T)) method. Methods 25: 402-408.

Lucchesi JC (1998). Dosage compensation in flies and worms: the ups and downs of X-chromosome regulation. Curr. Opin. Genet. Dev. 8: 179-184.

Lucchesi JC, Kelly WG and Panning B (2005). Chromatin remodeling in dosage compensation. Ann. Rev. Genet. 39: 615-651.

Marín I and Baker BS (2000). Origin and evolution of the regulatory gene male-specific lethal-3. Mol. Biol. Evol. 17: $1240-1250$.

Meeratana P and Sobhon P (2007). Classification of differentiating oocytes during ovarian cycle in the giant freshwater prawn, Macrobrachium rosenbergii (De Man). Aquaculture 270: 249-258.

Morales V, Regnard C, Izzo A, Vetter I, et al. (2005). The MRG domain mediates the functional integration of MSL3 into the dosage compensation complex. Mol. Cell. Biol. 25: 5947-5954.

Okuno T, Satou T and Oishi K (1984). Studies on the sex-specific lethals of Drosophila melanogaster. VII. Sex-specific lethals that do not affect dosage compensation. Jpn. F. Genet. 59: 237-247.

Palmer MJ, Mergner VA, Richman R, Manning JE, et al. (1993). The male specific lethal one (msl-1) gene of Drosophila melanogaster encodes a novel protein that associates with the X chromosome in males. Genetics 134: 545-557.

Park IS, Kim JH, Bang IC and Kim DS (1998). Histological study of the early gonadal development and sexual differentiation in Rhynchocypris oxycephalus. Dev. Reprod. 2: 69-74.

Qiao H, Fu HT, Jin SB, Wu Y, et al. (2012). Constructing and random sequencing analysis of normalized cDNA library of testis tissue from oriental river prawn (Macrobrachium nipponense). Comp. Biochem. Physiol. Part D Genomics Proteomics 7: 268-276.

Qiu GF, Yamanoa K and Unumaa T (2005). Cathepsin C transcripts are differentially expressed in the final stages of oocyte maturation in kuruma prawn Marsupenaeus japonicus. Comp. Biochem. Physiol. B Biochem. Mol. Biol. 140: 171-181.

Smith ER, Pannuti A, Gu WG, Steurnagel A, et al. (2000). The Drosophila MSL complex acetylates histone H4 at lysine 16, a chromatin modification linked to dosage compensation. Mol. Cell. Biol. 20: 312-318.

Sun MQ, Lin P, Chen Y, Wang YL, et al. (2012). Research advance of dosage compensation and MSL complex. Yi Chuan 34: 533-544.

Sural TH, Peng S, Li B, Workman JL, et al. (2008). The MSL3 chromodomain directs a key targeting step for dosage compensation of the Drosophila melanogaster X chromosome. Nat. Struct. Mol. Biol. 15: 1318-1325.

Tamura K, Dudley J, Nei M and Kumar S (2007). MEGA4: Molecular Evolutionary Genetics Analysis (MEGA) software version 4.0. Mol. Biol. Evol. 24: 1596-1599.

Zhang FY, Chen LQ, Wu P, Zhao WH, et al. (2010). cDNA cloning and expression of Ubc9 in the developing embryo and ovary of oriental river prawn, Macrobrachium nipponense. Comp. Biochem. Physiol. B Biochem. Mol. Biol. 155: 288-293.

Zhang YP, Fu HT, Qiao H, Jin SB, et al. (2013). Molecular cloning and expression analysis of transformer-2 gene during development in Macrobrachium nipponense (de Haan 1849). J. World Aquac. Soc. 4: 338-349.

Zhao GF, Li GL and Zhu CH (2009). Preliminary study on sex differentiation of Litopenaeus vannamei. J. Guangdong Ocean Univ. 29: 19-23.

Zhou SB, Yang YF, Maxwell JS, Antonio P, et al. (1995). Male-specific lethal 2, a dosage compensation gene of Drosophila, undergoes sex-specific regulation and encodes a protein with a RING finger and a metallothionein-like cysteine cluster. EMBO J. 14: 2884-2895.

Zhu CH, Xue HB, Li YJ, Huang GZ, et al. (2011). Effects of 4 nonylphenol on growth and sex differentiation in Macrobrachium rosenbergii. J. Fish. China 33: 365-371. 